\title{
Modeling and the main stages of spin coating process: A review
}

\author{
Haveen Ahmed Mustafa ${ }^{1}$, Dler Adil Jameel ${ }^{2 *}$ \\ ${ }^{I}$ Department of Physics, Faculty of Science, University of Zakho, Kurdistan Region, Iraq, havin1990@yahoo.com \\ ${ }^{2}$ Department of General Sciences, College of Basic Education, University of Zakho, Kurdistan Region, Iraq, \\ dler.jameel@uoz.edu.krd \\ *Correspondence: dler.jameel@uoz.edu.krd
}

\begin{abstract}
Spin coating is a technique employed for the deposition of uniform thin films of organic materials in the range of micrometer to nanometer on flat substrates. Typically, a small amount of coating material generally as a liquid is dropped over the substrate center, which is either static or spinning at low speed. The substrate is then rotated at the desired speed and the coating material has been spread by centrifugal force. A device that is used for spin coating is termed a spin coater or just a spinner. The substrate continued to spin and the fluid spins off the boundaries of the substrate until the film is reached the required thickness. The thickness and the characteristics of coated layer (film) are depending on the number of rotations per minute (rpm) and the time of rotation. Therefore, a mathematical model is obtained to clarify the prevalent method controlling thin film fabrication. Viscosity and the concentration of (solution) spin coating material are also affecting the thickness of the substrate. This article reviews spin coating techniques including stages in the coating process such as deposition, spin-up, stable fluid outflow (spin-off), and evaporation. Additionally, the main affecting factors on the film thickness in the coating process are reviewed.
\end{abstract}

Keywords: Spin-coating, modeling, stages of spin coating, thin film fabrication

Received: June 25 $5^{\text {th }}, 2021$ / Accepted: August $20^{\text {th }}, 2021 /$ Online: August $28^{\text {th }}, 2021$

\section{INTRODUCTION}

The researches for improvement of the variable electronic device are rapidly increasing for frequently applied in electronic maps, smart cards, optoelectronic applications (photovoltaic diode or light-emitting) and other applications. Thin film technology is considered an important character in nanotechnology, microelectronics and optics applications. In order to obtain thin film in either micro or nanoscale, commonly Physical Vapor Deposition (PVD), Chemical Vapor Deposition (CVD) and spin-coating techniques are used [1 - 3]. Spin coating is recognized as the most common technique that can be applied for the deposition of different materials in the form of solutions such as nanoparticles, polymers and biomaterials [4]. Also, it is the most convenient technique for fabricating hybrid solar with different thin film thicknesses. This is because in several instances the coating materials are polymeric and utilized in the form of a solution [5, 6]. Additionally, it is considered as an ideal technique to obtain very smooth, uniform and ultra-thin covering.
However, the disadvantage of this method is the difficulty of depositing materials on a large area of substrates [2]. The creative examination of the spin coating was realized by Emil et al. more than fifty years ago [7]. The authors measured the spreading of a thin axisymmetric film of Newtonian fluid on a planner substrate that rotates with constant angular speed [7]. Fu et al. used spin coating to produced superhydrophobic transparent coat [8]. High transparent zinc oxide ( $\mathrm{ZnO})$ thin film successfully has been dropped on the glass substrate by using the spin coating process [9]. K. M. Zaidan [10] used spin speeds in the range of 1000-5000 rpm to fabricate thin films of PANI prepared on n-type Si substrates with PANI thickness of $30 \mathrm{~nm}$. Hybrid solar cells have been manufactured by depositing organic polymer polyaniline (PANI) over inorganic semiconductor gallium arsenide ( $\mathrm{GaAs}$ ) substrates using the spin coating technique [11]. In addition, Jameel et al. [12] utilized spin coating to deposit organic polymer PANI on GaAs substrates with different orientations. Moreover, the effect of the rotation number of spin coating on the thickness of hybrid solar cell devices was studied by Mustafa et al. [13]. The authors obtained that the 
thicknesses of the deposited PANI layer over GaAs are $58 \pm 2$ $\mathrm{nm}, 58 \pm 2 \mathrm{~nm}$, and $54 \pm 2 \mathrm{~nm}$ for sample spinning at 2000, 3000, and $4000 \mathrm{rpm}$, respectively [13]. Furthermore, it has been determined that the number of rotations has a considerable effect on the thickness of the coating layer and the efficiency of PANI/TiO2/FTO-Glass Photoanode has been studied [14].

This work reviews and explain the concept behind the spin coating system and its applications. Additionally, the process and four steps, which are called deposition, spin-up, stable fluid outflow and evaporation of the spin coating system are reviewed. Furthermore, we presented the effects of spin speed, acceleration and rotating time on the thickness of thin films fabricated by the spin coating technique.

\section{KEY STAGES OF SPIN COATING}

In general, the dynamics of spin coating can effectively be demonstrated by separating the complete process into four stages; deposition, spin-up, spin-off and evaporation of solvents, as shown in Fig. 1 [14]. The first three steps are commonly taking place consecutively. However, the fourth step occurs through the entire preparation process [1]. The third step (flow controlled) and fourth step (evaporation controlled) are very important because they have the greatest effects on final coating thickness.

\section{A. Deposition}

In this step, the substrate is placed on the spin coater machine and the coated material as a solution is dropped on the substrate surface, as illustrated in Fig. 1(a). The substrate is either not rotating called static deposition or rotating at a low speed called dynamic deposition [15]. In the static deposition, some drops of coating material depend on the size of the substrate and the viscosity of the coating solution are dropped on the substrates [16]. On the other hand, in the dynamic deposition stage, the material has been dispensed while the substrate is rotating at low rotations typically around $500 \mathrm{rpm}$ since it serving in less wastage of coating solution [16]. Dispersion of the coating material takes place as a result of centrifugal force and height is decreased to critical height.

\section{B. Spin-up}

After the deposition of materials the substrate will begin to spin until it reaches a certain speed. This step is typically regarded as destructive fluid ejection from the surface of substrate by the rotational process [17]. The centrifugal force that results from the acceleration and angular speed applied to the substrate cases, the flow of fluid outward the substrate surface, as displayed in Fig. 1(b). The thickness of the coated layer is strongly affected by the spin speed and rotating time of the spin coater.

\section{Spin-off}

This stage is considered as the third stage among the spin coating technique. It is started once the wafer is spinning at a continuous ratio and liquid viscous forces control solution thinning conduct, which is also called stable solution thinning. Through this step, the fluid spreads out through the surface and at that time the edge of the substrate is removed as the film thins out, as displayed in Fig. 1(c). Effects of edge are commonly observed since the liquid runs homogeneously obvious, however should create droplets at the border to be thrown off. As a result, the thickness of thin film depends on the surface tension, viscosity and rotation rate. There might be a small droplet of coating thickness difference around the edge of the final wafer [18].

\section{Evaporation}

This is the final step in the deposition process of the spin coating technique. It is occurred during the entire procedure of film construction and results from the high rotation of the spin coater [19]. In this phase, the substrate spin at a steady rate and the coating thinning conduct is dominated by solvent evaporation. It is important to point out that the thicker film will be obtained if the time of the evaporation process is increased, which means increasing the concentration and viscosity of the solution. Therefore, the solution must evaporate immediately at room temperature. (a)

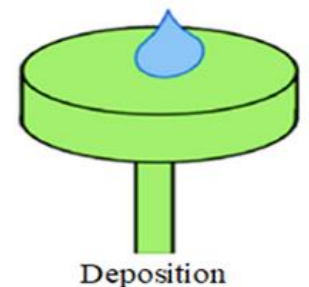

(c)

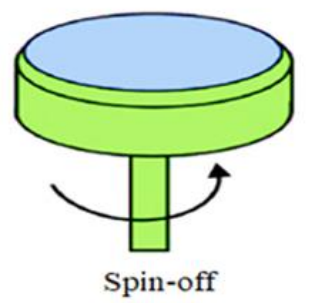

(b)

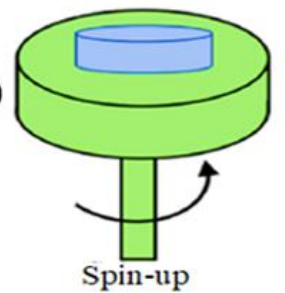

(d)

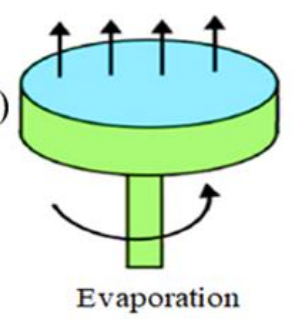

Fig. 1. Four stages of spin coating process

\section{THE AFFECTED FACTORS ON THIN FILM THICKNESS IN COATING PROCESS}

Factors that impact on the thickness of the thin film of materials coated on the substrates, these four cases will be discussed below.

\section{A. Spin Speed}

Spin speed can be considered as one of the significant factors in the spin coating system $[20,21]$. The speed of spin is measured by rotation per minute (rpm). The speed (rpm) of the substrate impacts the degree of radial (centrifugal) force applied to the liquid resin and the characteristic as well as velocity of the air directly above it. Particularly, the final film thickness is determined generally by the high-speed spin step. In this step, comparatively slight differences of $\pm 50 \mathrm{rpm}$ could produce a change of about $10 \%$ of the resulting thickness. The thickness of a film is commonly an equilibrium between the force employed to shear the liquid resin on the way to the substrate edge and the drying ratio, which influences the resin viscosity [20]. 


\section{B. Acceleration}

The wafer acceleration a across the ending speed could be described as the second important factor which influences the properties of the coated film [22]. Subsequently, the resin starts to dry throughout the first part of the spin series, and it is very essential to precisely control acceleration. In the first few seconds of some process, around $50 \%$ of the solvents in the resin will be wasted by evaporation. Additionally, the acceleration factor plays an important role in the properties of the fabricated sample in the spin coating process. The spinner acceleration is able to be programmed with a resolution of $1 \mathrm{rpm} / \mathrm{second}$ [20].

\section{Rotating Time}

Time can be considered as another important aspect that affects the spin coating procedure and the characteristic of the final film $[22,23]$. The degree of drying of the liquid resin through the spin method is well- expressed by the nature of the liquid itself (volatility of the solvent systems applied) also by the air that surrounded the sample throughout the spin progress. In addition, there are also other factors such as temperature and humidity that have a considerable impact on the characteristics of the produced thin film. It is also identical to minimize the airflow and related confusion over the wafer itself, or at least stayed constant, thru the spin method.

\section{MODELING THE SPIN COATING PROCEDURE}

Theoretical study of spin coating has not been initiated in a long time, although it has been used in the industry of semiconductors. The precise theory study of spin coating entitles preferable design and control of the operation in its different applications. For the theoretical study, supposed utilizing cylindrical polar coordinates $(z, r$ and $\theta)$ with origin at the center of rotation, axes $\theta$ and $r$ rotating with the plane with angular velocity $\omega$, and $z$ perpendicular to the plane. However, for Newtonian fluid, the balance between centrifugal forces per unit volume and viscous is yielded by [24]:

$$
-\eta=\left[\frac{d^{2} v}{d Z^{2}}\right]=\rho r \omega^{2}
$$

where $\eta$ is absolute viscosity, $v$ is velocity in the direction of $r$ and $\rho$ is fluid density.

By using boundary condition $\frac{\partial v}{\partial z}=0$ at $z=h$ gives

$$
V=\frac{1}{\eta}\left(\rho \varpi^{2} r h z-\frac{1}{2} \rho \varpi^{2} r h z^{2}\right)
$$

The radial flow $q$ per unit length of the circumference is expressed by the following equation

$$
q=\int_{0}^{h} v(z) d z=\frac{\rho \omega^{2} r h^{2}}{3 \eta}
$$

The equation for continuity is yielded by

$$
r\left[\frac{\partial h}{\partial t}\right]=-\frac{\partial(r q)}{\partial r}
$$

By substituting Eq. (3) in Eq. (4) one can get the following equations.

$$
\frac{\partial h}{\partial t}=-K\left(\frac{1}{r}\right) \frac{\partial\left(r^{2} h^{3}\right)}{\partial r}
$$

where $K=\frac{\rho \omega^{2}}{3 \eta}$.

The especial solution of eq. (4), before taking a general solution, is shown as below. This equation is depending on time factor only.

The special solution is given by

$$
\frac{\partial h}{\partial t}=3 K r h^{2} \frac{\partial h}{\partial r}-2 K h^{3}
$$

Since at the beginning the film is homogenous, $h$ is not depending on $r$ and therefore $\frac{\partial h}{\partial r}=0$ that yields

$$
\frac{\partial h}{\partial t}=-2 K h^{3} \text { or } \frac{\partial h}{h^{3}}=-2 K \partial t
$$

By integrating Eq. (7) at boundaries conditions at $t=0, h=h_{0}$ and at $\mathrm{t}, h=h_{t}$ then one gets

$$
h_{t}=\frac{h_{0}}{\sqrt[2]{1+4 h_{0}^{2} K t}}=\frac{h_{0}}{\sqrt[2]{1+\frac{4 h_{0}^{2} \rho \omega^{2} t}{3 \eta}}}
$$

The general solution is expressed by connecting total and partial derivatives as following

$$
\frac{d h}{d t}=\frac{\partial h}{\partial t}+\frac{\partial h}{\partial r} \frac{d r}{d t}
$$

where $\frac{\partial h}{\partial t}=-2 K h^{3}$ and $\frac{d r}{d t}=3 K r h^{2}=\frac{3 K r h_{0}^{2}}{1+4 h_{0}^{2} K t}$

$$
\begin{aligned}
& \Rightarrow \quad \ln \left(\frac{r}{r_{0}}\right)=\frac{3}{4} \ln \left(1+4 h_{0}^{2} K t\right)=\ln \left(1+4 h_{0}^{2} K t\right)^{3 / 4} \\
& \Rightarrow \quad r=r_{0}\left(1+4 h_{0}^{2} K t\right)^{3 / 4}
\end{aligned}
$$

By considering the Gaussian initial contour of the formula

$$
h_{0}=a e^{-a^{2} r_{0}^{2}} \Rightarrow \frac{h_{0}}{a}=e^{-a^{2} r_{0}^{2}}
$$

For Gaussian plus uniform initial contour of the form

$$
\frac{h_{0}}{a}=e^{-a^{2} r_{0}^{2}}
$$

the exponential expression will conduct to levelling with the rise in $t$.

It is important to mention that the evaporation stage plays a significant role in the final thickness of the film. Therefore, the 
evaporation term as a constant to the thinning rate has been added, as presented below

$$
\frac{d h}{d t}=-2 K h^{3}-e
$$

where $e$ is evaporation rate $\left(\mathrm{ml} \mathrm{s}^{-1} \mathrm{~cm}^{-1}\right)$. When the viscous flow rate and evaporation rate become equal at the transition point, evaporation depends on rotation rate $(\omega)$.

$$
e=C \sqrt{\omega}
$$

$\mathrm{C}$ is constant and $h_{f}$ refers to the thickness of the final film, then by taking cube root and reordering:

$$
h=(2 K)^{-1 / 3}\left(-\frac{d h}{d t}\right)^{1 / 3}-h_{f}
$$

The flow constant and the thickness of the final film are found from the slope and the intercept, respectively.

The solution height $h$ can be divided into the solvent height $L$ and the solid height $S$, as is presented below.

$$
h=L+S \text {. }
$$

The solid concentration $\mathrm{C}(\mathrm{t})$ is given as $C(t)=\frac{S}{(S+L)}$. Thus, from the equation of continuity it can be obtained the following formula

$$
\frac{d S}{d t}=-C(t) \frac{2 \omega^{2} h^{3}}{3 \vartheta}
$$

where $\vartheta=\frac{\eta}{\rho}, \vartheta$ represents to the kinetic viscosity. The change of height of liquid with including evaporation rate $\varphi$ of the liquid is given by

$$
\frac{d S}{d t}=[-1+C(t)] \frac{2 \omega^{2} h^{3}}{3 \vartheta}-\varphi
$$

The final film height can be presented by

$$
h=\left[\frac{3 \vartheta_{0} \varphi C^{3}(t)}{2 \omega^{2}\left\{1-C_{0}(t)\right\}}\right]^{1 / 3} \text { or } h \propto \omega^{-2 / 3}
$$

\section{ADVANTAGES}

There are many advantages of a spin coating system in coating operations. Some advantages are shown below [24][25]:

1. The biggest advantage is easily changing the film thickness by changing spin speed or changing viscosity photoresist of materials. However, with the different coating system, many have several fixed parameters, making control of coating extra complex.

2. The additional benefit of this technique is that the thin layers can be deposited quickly and easily as well as more uniform.
3. It is a fast functional system and low price.

4. It is a relatively low cost comparing physical vapor deposition and other methods.

5. Another advantage of spin coating is less loss of materials than of other systems [24][25].

\section{DiSADVAnTAgeS}

There are a few disadvantages of spin coating process. The main drawback of this process, it cannot be used for large substrates because large substrates are unable to weave at a appropriately high rate to let the film be thin. Moreover, it is difficult to create multilayer structures ( $>2$ layers) and control accurately the deposition (homogeneity, rugosity, etc.). Furthermore, this system is incapable creates thin film less than $10 \mathrm{~nm}[24]$.

\section{APPLICATIONS}

Spin For many decades spin coating system has been utilized as the main technique for the application of thin films. Besides its application in the thin film field, it is also extensively employed in the fabrication of major electronic devices here is some applications of the spin coating process [2, 23]:

1. The device of solar cells.

2. Detectors.

3. Integrated circuits

4. Magnetic disk for data storage.

5. VLSI (very large-scale integration).

6. Optical mirrors.

7. Sensors.

8. Nanoscale device (quantum dots, carbon nanotubes).

9. Photoresist for designing wafers in microcircuit manufacture.

10. DVD and CD ROM.

11. Flat-screen display coatings.

12. Insulating films for microcircuit production for example polymers (where it can be employed to manufacture films with a thickness less than $10 \mathrm{~nm}$ ).

13. Conductive oxide and antireflection coatings etc. [2, 23].

\section{SPIN COATER}

A spin coater can be considered as a main device that is generally used in spin coating process. It defined an ideal device for drying the substrate or deposition of fluid on the surface of substrate in order to produce thin film. There are many different types and modules available. Fig. (2) shows the high speed (1200) rpm Spin coater model WS-650MZ23NPPB. 


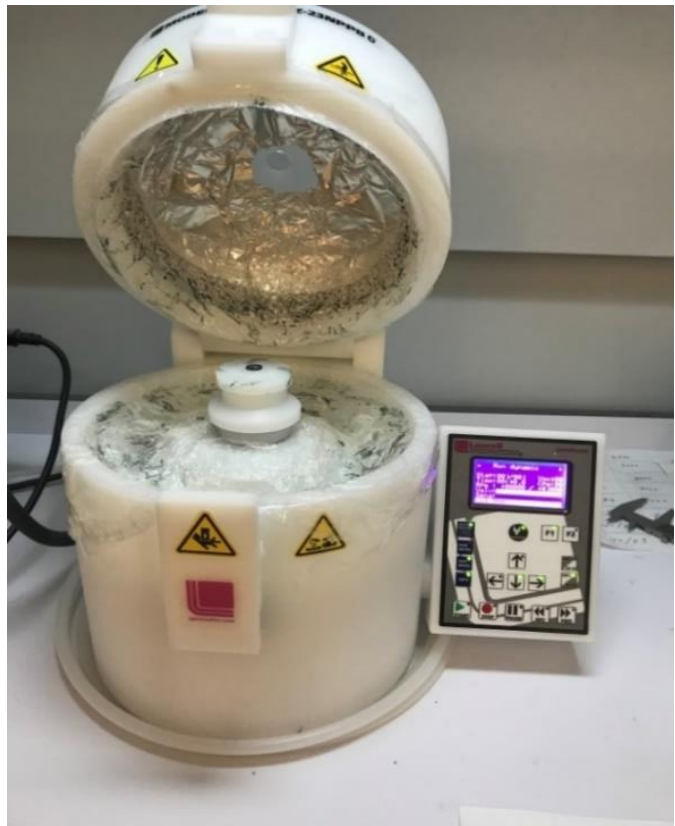

Fig. 2. Spin coater model WS-650MZ-23NPPB

\section{CONCLUSIONS}

This review presented the fundamental physics concepts behind the spin coating procedure. Also, it describes the stages that take place during the procedure of fabrication of thin films. The stages are including deposition (static and dynamic deposition), spin up, stable fluid outflow and evaporation. It is revealed that in this technique the thickness of the coating layer can be controlled by controlling the spin speed time and viscosity of the coating material. Moreover, a mathematical model of a spin coating system has been consequent to illustrate the predominant mechanism controlling layer fabrication. It results in the relation between the thickness of thin-film and time. The thickness remarkably decreases with increasing the $\mathrm{rpm}$ and increasing spinning time. Advantages and drawbacks, as well as some industrial applications, are debated.

\section{REFERENCES}

[1] J.A. Mera-Córdoba, M.A. Mera-Córdoba, C.A. Córdoba-Barahona, Spin Coating technique for obtaining nanometric thin films in the system La0.7Sr0.3MnO3, Rev. Fac. Ing. $26 \quad$ (2017) 123. https://doi.org/10.19053/01211129.v26.n44.2017.5783.

[2] A. Mishra, N. Bhatt, A.K. Bajpai, Nanostructured superhydrophobic coatings for solar panel applications, Elsevier Inc., 2019. https://doi.org/10.1016/B978-0-12-815884-5.00012-0.

[3] D.A. Jameel, Thin Film Deposition Processes, Int. J. Mod. Phys. Appl. 1 (2015) 193-199.

[4] A. Salehi, P. Naderi, F. Boroumand, A. Dunbar, Fabrication and Characterization of Hybrid Photovoltaic Devices Based On N-Type GaAs and Polymer Composites, 2018. https://doi.org/10.11159/ehst18.116.

[5] P. Kaienburg, B. Klingebiel, T. Kirchartz, Supporting Information for Spin-coated planar Sb $2 \mathrm{~S} 3$ hybrid solar cells reaching $5 \%$ efficiency Additional Figures and Tables, (n.d.) 1-6.

[6] K. Wantana, P. Aniwat, S. Bunlue, T. Alongkot, K. Anusit, K. Pisist, Study of thin film coating technique parameters for low cost organic solar cells fabrication, Mater. Today Proc. 4 (2017) 6626-6632. https://doi.org/10.1016/j.matpr.2017.06.177.

[7] A.G. Emslie, F.T. Bonner, L.G. Peck, Flow of a viscous liquid on a rotating disk, J. Appl. Phys. 29 (1958) 858-862. https://doi.org/10.1063/1.1723300.

[8] Z. He, M. Ma, X. Lan, F. Chen, K. Wang, H. Deng, Q. Zhang, Q. Fu, Fabrication of a transparent superamphiphobic coating with improved $\begin{array}{lllll}\text { stability, } & \text { Soft } & \text { Matter. } 7 & \text { (2011) 6435-6443. }\end{array}$ https://doi.org/10.1039/c1sm05574g.

[9] M. Dahnoun, A. Attaf, H. Saidi, A. Yahia, C. Khelifi, Structural, optical and electrical properties of zinc oxide thin films deposited by sol-gel spin coating technique, Optik (Stuttg). $134 \quad$ (2017) 53-59. https://doi.org/10.1016/j.ijleo.2017.01.014.

[10] K.M. Zaidan, H.F. Hussein, R.A. Talib, A.K. Hassan, Synthesis and characterization of (PAni/n-Si)solar cell, Energy Procedia. 6 (2011) 8591. https://doi.org/10.1016/j.egypro.2011.05.010.

[11] D.A. Jameel, Electrical performance of organic/inorganic hybrid solar cell devices based on n-type GaAs substrate orientations and a conjugated polymer (PANI), Appl. Phys. A. 127 (2021) 1-10.

[12] D.A. Jameel, J.F. Felix, M. Aziz, N. Al Saqri, D. Taylor, W.M. De Azevedo, E.F. Da Silva Jr, H. Albalawi, H. Alghamdi, F. Al Mashary, M. Henini, High-performance organic/inorganic hybrid heterojunction based on Gallium Arsenide (GaAs) substrates and a conjugated polymer, Appl. $\begin{array}{lllll}\text { Surf. } & \text { Sci. } & 357 & \text { (2015) }\end{array}$ https://doi.org/10.1016/j.apsusc.2015.09.209.

[13] H.A. Mustafa, D.A. Jameel, H.I. Salim, S.M. Ahmed, The Effects Of NGaAs Substrate Orientations on The Electrical Performance of PANI/NGaAs Hybrid Solar Cell Devices, Sci. J. Univ. Zakho. 8 (2020) 149-153. https://doi.org/10.25271/sjuoz.2020.8.4.773.

[14] S.W. Himmah, U. Sa'Adah, A.D. Iswatin, M. Diantoro, A. Hidayat, Z.A.I. Supardi, The Effect of Spin Coating Rotation on the Optoelectronic Properties of PANI/TiO2/FTO-Glass Photoanode, IOP Conf. Ser. Mater. Sci. Eng. 515 (2019). https://doi.org/10.1088/1757-899X/515/1/012084.

[15] M.D. Tyona, A theoritical study on spin coating technique, Adv. Mater. Res. 2 (2013) 195-208. https://doi.org/10.12989/amr.2013.2.4.195.

[16] S.L. Hellstrom, Published course work for physics 210, (2007).

[17] D.B. Mitzi, L.L. Kosbar, C.E. Murray, M. Copel, A. Afzali, Highmobility ultrathin semiconducting films prepared by spin coating, Nature. 428 (2004) 299-303. https://doi.org/10.1038/nature02389.

[18] D.A.H. Hanaor, G. Triani, C.C. Sorrell, Morphology and photocatalytic activity of highly oriented mixed phase titanium dioxide thin films, Surf. Coatings Technol. 205 (2011) 3658-3664.

[19] T. Peeters, B. V Remoortere, Parameters of the spin coating process, J. Appl. Polym. Sci. 12 (2008) 234-239.

[20] G. Demircan, E.F. Gurses, A. Acikgoz, S. Yalcin, B. Aktas, Effects of spin coating parameters on stress, electrical and optical properties of multilayer $\mathrm{ZnO}$ thin film prepared by sol-gel, Mol. Cryst. Liq. Cryst. 709 (2020) 61-69.

[21] D. Mao, G. Lv, G. Gao, B. Fan, Fabrication of polyimide films with imaging quality using a spin-coating method for potential optical applications, J. Polym. Eng. (2019). https://doi.org/10.1515/polyeng2019-0177.

[22] M. Sbeta, A. Atilgan, A. Atli, A. Yildiz, Influence of the spin acceleration time on the properties of $\mathrm{ZnO}$ : Ga thin films deposited by sol-gel method, J. Sol-Gel Sci. Technol. 86 (2018) 513-520.

[23] S. B. W., H. N. Narasimha Murthy, M. Krishna, S. C. Sharma, Investigation of Influence of Spin Coating Parameters on the Morphology of $\mathrm{ZnO}$ Thin Films by Taguchi Method, Int. J. Thin Film. Sci. Technol. 2 (2013) 143-154. https://doi.org/10.12785/ijtfst/020210.

[24] N. Sahu, B. Parija, S. Panigrahi, Fundamental understanding and modeling of spin coating process: A review, Indian J. Phys. 83 (2009) 493-502. https://doi.org/10.1007/s12648-009-0009-z.

[25] A. Boudrioua, M. Chakaroun, A. Fischer, Organic Light-emitting Diodes, Org. Lasers. (2017) 49-93. https://doi.org/10.1016/B978-1-78548-158$1.50002-\mathrm{X}$ 